\title{
ESTRATEGIAS DE AFRONTAMIENTO Y MIEDO A HABLAR EN PÚBLICO EN ESTUDIANTES UNIVERSITARIOS A NIVEL DE GRADO
}

\section{COPING STRATEGIES AND PUBLIC SPEAKING FEAR AMONG UNDERGRADUATE STUDENTS}

\author{
Iván Maldonado \\ Universidad Católica del Uruguay \\ Micaela Reich \\ Universidad Católica del Uruguay
}

\begin{abstract}
Resumen: En Uruguay son escasas las investigaciones científicas que estudian la relación entre Miedo a Hablar en Público y Afrontamiento, y menos las que estudian el papel de variables socio-demográficas. Este estudio tuvo como objetivo describir y comparar los niveles de Miedo o Ansiedad de Hablar en Púbico (CASO-A30) y las Estrategias de Afrontamiento (Brief COPE) de los estudiantes de 2do. y 4to. año a nivel de grado y la incidencia de variables socio-demográficas (Encuesta elaborada ad hoc para este estudio). La muestra analizada $(n=76), 34$ alumnos de 2 do. año y 42 alumnos de 4 to., se compuso mayoritariamente por mujeres $(88,2 \%)$ de entre 21 y 25 años $(57,9 \%)$ sin actividad laboral $(61,8 \%)$; aportó información por medio de cuestionarios de auto-informe, hallándose correlaciones negativas y positivas estadísticamente significativas entre las variables dependientes y algunas socio-demográficas. Se concluyó que las mujeres de entre 21 y 25 años, cursando 2 do. año y sin actividad laboral evidenciaron ser la población de mayor vulnerabilidad.
\end{abstract}

Palabras Clave: Miedo de hablar en público, afrontamiento, estrés, estudiantes universitarios, fobia social

\begin{abstract}
A growing, yet limited body of scientific literature addresses the relationship between Public Speaking Fear, Coping Strategies, and demographic variables in Uruguay. The aim of this study was to examine the association between Public Speaking Fear/Anxiety (CASO-A30), Coping Strategies (Brief COPE), and demographic factors. Participants included a total of 76 undergraduate students in 2 nd $(n=34)$ and 4 th year $(n=42)$, respectively. The sample was composed by a majority of female participants $(88,2$ $\%$ ), aged $21-25$ years $(57,9 \%)$, and unemployed $(61,8 \%)$ at the time of data collection. Data were assessed utilizing self-report questionnaires. The results yielded several statistically significant associations between demographic and psychological factors. Results suggest that higher vulnerability was indicated by being female, in 2nd year, unemployed and aged 21-25 years.
\end{abstract}

Keywords: Public speaking fear, coping, stress, undergraduate students, social phobia

\section{Introducción}

Actualmente, en nuestro país existe un escaso número de estudios realizados sobre la relación existente entre Miedo a Hablar en Público (en adelante MHP) y Estrategias de Afrontamiento en población universitaria. Menor aún es el número de trabajos que vinculan estas variables a factores socio-demográficos. Sin embargo, las publicaciones realizadas en otros países informan acerca de una alta incidencia de MHP en la población universitaria (Henderson \& Pollard, 1988; Kozak, Lang, Levin, \& Mliller 1983, Borkovec \& Lane, 1984; Fisher, 1984, 1994;
Bados, 1992; Hernández, Polo y Pozo, 1996; Gutiérrez, 1996; Betson, Lam, Stewart, Wong, \& Wong, 1999; Alvarado, García y Jiménez, 2000; D'El Rey y Pacini, 2005); pudiendo esto conllevar a una repercusión negativa en la vida profesional y académica Bados (1992), Alvarado et al. (2000); así como en la población general, Bados (1992); Fernández-Turrado, Herrero, Orejudo, Nuño y Ramos (2005). Considerando estos antecedentes entendemos es relevante caracterizar y describir MHP y su relación con Estrategias de Afrontamiento en esta muestra de estudiantes universitarios uruguayos. 
Lazarus (2000) define el Afrontamiento como los esfuerzos cognitivos y conductuales en constante cambio para la resolución de las demandas internas y/o externas específicas que son valoradas como impositivas o excesivas para los recursos de las personas. Por su parte, Fernández-Abascal (1997) define los Estilos de Afrontamiento como las predisposiciones personales para hacer frente a situaciones, siendo los responsables de las preferencias individuales por el uso de uno u otro tipo de Estrategia de Afrontamiento, así como de la estabilidad temporal y situacional de las mismas, mientras que las Estrategias son definidas por este autor como los procesos concretos y cambiantes que se utilizan en cada contexto dependiendo del desencadenante.

Partimos de la base de que Hablar en Público, es considerado tanto por adultos como por adolescentes, una de las situaciones sociales más difíciles (Argyle, 1981; Brown \& Harris, 1982). Estas situaciones percibidas cursan con niveles elevados de Ansiedad, y adquieren una significación clínica cuando aumentan hasta tal punto en que repercuten negativamente en el ámbito personal, laboral, académico, familiar y social de la vida cotidiana de la persona (Echeburúa, 1993). Cuando las demandas externas se tornan excesivas y las herramientas internas se perciben como escasas e ineficientes, las respuestas de Ansiedad adquieren una mayor magnitud y se vuelven exageradas, lo que podría provocar un malestar significativo para la persona.

Algunos autores clásicos como Beck (1976), conceptualizan el MHP como un conjunto de creencias disfuncionales, sesgos cognitivos y pensamientos irracionales (Clark \& Wells, 1995), que afectan la atención de la persona, sesgando la información acerca de sí mismo y de su entorno. El MHP se trata de una respuesta de Ansiedad frente a determinados estímulos. En esta respuesta intervienen tanto componentes biológicos y genéticos (Beatty \& Valencic, 2000; Keaten, \& Kelly, 2000), como procesos de modelado y refuerzo provenientes del entorno escolar y familiar (Duarte et al., 2002). Sin embargo, otros autores (Beatty \& Valencic, 2000) insisten en un déficit de habilidades sociales como una de las causas principales del temor o la Ansiedad experimentados por las personas frente a situaciones en las que deben Hablar en Público. Bados (2001), remarca que el MHP implica tres sistemas de respuesta (cognitivo, motor y autónomo), los cuáles interactúan entre sí y la aparición de estos componentes está sujeta a estímulos externos (socio-ambientales) e internos (cognitivos y fisiológicos).

El Modelo Transaccional del Estrés y Afrontamiento propuesto por Folkman y Lazarus (1984), es el más utilizado por la literatura en el estudio del MHP. Este Modelo propone que Afrontar el MHP es un proceso que se verá influido tanto por factores internos y por variables motivacionales de la persona (Martin, Mottet, \& Myers, 2004); por factores de orden biológico y genético (Beatty \& Valencic, 2000; Keaten \& Kelly, 2000); por las experiencias de modelado obtenidas desde el entorno escolar y familiar (Duarte et al., 2002); por el déficit de habilidades sociales al momento de enfrentarse a situaciones de exposición pública (Bados, 2008; Beatty \& Valencic, 2000); las reacciones de la audiencia (McAnintrye \& McDonald, 1998); la presencia de comunicadores compulsivos como interferencia (Fortney, Jonson, \& Long, 2001); los procesos de feedback o devoluciones realizadas por profesores o iguales (King \& Smith, 2004); y el estilo comunicativo (Martin et al., 2004). El rol de los educadores también parece desempeñar un papel importante, en especial, el grado de cercanía expresado hacia los alumnos (Ellis, 1995). El grado de conocimiento de los pares, así como el tamaño de la audiencia o el lugar desde donde se realiza la exposición también son determinantes que influyen en la manera en cómo se vivencia la experiencia de Hablar en Público (Booth-Butterfierld, 1988).

\section{Variables socio-demográficas}

- Nivel educativo. En relación a las variables socio-demográficas, se han encontrado trabajos científicos que indican una correlación negativa entre el nivel educativo de las personas y la Ansiedad que presentan (Casullo y Fernández, 2001). En estudiantes universitarios (Heinrich \& Spielberg, 1982), entre quienes las diferencias en aptitud intelectual son habitualmente menores, la mayoría de los estudios muestran una ausencia de relación. Teniendo en cuenta los trabajos realizados con personas que tenían un bajo nivel de estudios alcanzado y comparando los resultados con los hallados en poblaciones universitarias, podría interpretarse que la Ansiedad perjudica menos a medida que aumenta el nivel intelectual (Heinrich \& Spielberg, 1982). 
- Género. El género es otra de las variables socio-demográficas en base a la cual la literatura señala diferencias significativas respecto al MHP (e. g., Bados, 1992; Forde, Stein, \& Walker, 1996; Fernández-Turrado et al., 2005; Caballo, Arias, Equipo de Investigacion CISO-A, Irurtia y Salazar, 2010) quienes describen mayores niveles de Ansiedad entre las mujeres. Se encontró un consistente cuerpo de literatura científica que señala a la adolescencia como etapa en la que suele desarrollarse el MHP con mayor frecuencia (Argyle, 1981; Brown \& Harris, 1982; Bird et al.,1987; Bland, Newman, \& Orn,1988; Fyer, Gorman, Martin, Liebowitz, \& Schneier,1992; Angst \& Degonda, 1993; Davidson et al.,1993; Echeburúa 1993; Ehlers, Hofmann, \& Roth,1995; Bersabé, Bragado, Carrasco y Sánchez, 1996; Forde et al.,1996; Bascarán, Iglesias, Jiménez y Sáiz, 1999; Alfano, Beidel, Ferrell, \& Yeganeh,2001; Hidalgo, Inglés y Méndez, 2002; Caballo, GarcíaLópez, López-Gollonet, Olivares y Rosa 2003; Fernández-Turrado et al., 2005). Por su parte, los resultados encontrados por Caraveo y Colmenares (2000), indicarían un comienzo de los síntomas entorno a los 24 años para las mujeres. Se deduce de esta bibliografía que si bien el género femenino evidenciaría mayores niveles de Ansiedad registrada en comparación al género masculino, las mujeres desarrollarían mayores niveles de Ansiedad hacia la adolescencia tardía o juventud a diferencia de los hombres quienes indicarían mayores niveles de Ansiedad antes de los 20 años.

- Actividad laboral. Bermúdez, Ciccottelli, García, Gascón y Olmedo (2003), realizaron un trabajo científico en el que comparaban personas laboralmente activas y personas desocupadas. Este estudio señala que quienes se encontraban desocupados informaban mayores niveles de quejas somáticas, menor calidad de vida, menores niveles de satisfacción general y mayores niveles de Estrés diario que las personas que se encontraban laboralmente activas.

\section{Estrategias, Estilos de Afrontamiento y variables socio-demográficas}

- Estrategias de Afrontamiento, nivel educativo y edad. Según Contini, Fernández, De la Iglesia, Organato y Savedra (2009), a medida que aumenta la escolarización de las personas aparece una mayor tendencia a elegir un Afrontamiento más centrado en resolver el problema. En este sentido, Casullo y Fernández (2001) hallaron que conforme aumentaba la edad se observaba la utilización de Estrategias de Afrontamiento menos evitativas y más dirigidas a resolver el problema.

- Estrategias de Afrontamiento y género: Los trabajos realizados por Guevremont, Spirino, Stark y Williams (1989); Bird y Harris (1990); Frydenberg y Lewis (1991, 1993, 1999); Bolognini y Plancherel (1995); Copeland y Hess, (1995); Zeidner (1995); Bolognini, Halfon y Plancherel (1998); Frydenberg, Parsons y Poole (1996); Noam y Recklitis (1999); Washburn (2000) y Maslach y Reevy (2001), evidenciaron una tendencia de las mujeres a utilizar Estrategias de Afrontamiento Emocionales en mayor medida que los hombres, mientras que éstos últimos reportaron una mayor utilización de Estrategias dirigidas a resolver el problema (Nolen-Hoeksema,1994). Este autor encontró que las mujeres serían más proclives a rumiar frente a los problemas, y los hombres tenderían a actuar ante una dificultad que se les presenta.

- Estrategias de Afrontamiento y actividad laboral: Según lo planteado por Bermúdez et al. (2003), las personas que se encuentran laboralmente activas perciben un mayor grado de control sobre las situaciones estresantes que quienes no trabajan, estando ello asociado a dirigir sus Estrategias de Afrontamiento en mayor medida hacia tomar una postura activa ante las situaciones estresantes, y menos hacia la evitación del problema. Cabe mencionar que dentro de las relaciones estadísticamente significativas encontradas por Bermúdez et al. (2003), las Estrategias de Afrontamiento destinadas a evitar el estresor se hallaron asociadas con mayores niveles de síntomas somáticos entre las personas laboralmente desocupadas.

Asimismo, tanto el Afrontamiento Evitativo como el Emocional correlacionaron positivamente con un mayor deterioro del sistema inmune según estos autores.

Bermúdez et al. (2003), encontraron que el Afrontamiento basado en el apoyo social presentó una correlación negativa con los síntomas somáticos, con el Afrontamiento Evitativo, y positiva con la calidad de vida.

Partiendo de los datos encontrados en la literatura científica que plantean relaciones entre las variables socio-demográficas y los niveles de MHP, así como en relación a los Estilos y Estra- 
tegias de Afrontamiento, surgen las siguientes hipótesis:

Hipótesis 1: Considerando los trabajos de Heinrich y Spielberg (1982); Casullo y Fernández (2001), se observará una relación significativa entre el nivel de estudios alcanzado por los estudiantes y el grado de MHP detectado, así como las Estrategias de Afrontamiento que utilicen.

Hipótesis 2: En base a los resultados obtenidos por Contini et al. (2009), conforme sea mayor el nivel de estudios, se observará una tendencia hacia la elección de Estrategias de Afrontamiento preferentemente centradas en resolver el problema.

Hipótesis 3: A partir de los estudios realizados por Casullo y Fernández (2001), conforme aumente la edad se observarán Estilos de Afrontamiento menos evitativos y más dirigidos a resolver el problema.

Hipótesis 4: Siguiendo como referencia los trabajos de varios científicos ( Argyle, 1981; Brown \& Harris,1982; Bird et al.,1987; Bland et al.,1988; Fyer et al.,1992; Angst \& Degonda, 1993; Davidson et al., 1993; Echeburúa, 1993; Elhers et al.,1995; Bersabé et al., 1996; Forde et al.,1996; Bascarán et al., 1999; Alfano et al., 2001; Hidalgo et al., 2002; Caballo et al., 2003; Fernández-Turrado et al., 2005), los estudiantes cuyas edades se sitúen entre los 19 y 20 años presentarán mayores grados de MHP en comparación al resto de las franjas etarias.

Hipótesis 5: Partiendo de lo sugerido por Heinrich y Spielberg (1982) y Casullo y Fernández (2001), los niveles de MHP serán menores para los alumnos de 4 to que para los de 2 do.

Hipótesis 6: Según los datos encontrados por Caballo et al. (2010); Bados (1991); Forde et al. (1996); Fernández-Turrado et al. (2005), se observaran diferencias en los grados de MHP según el género. Las mujeres obtuvieron en estos estudios puntuaciones que indicaban mayores niveles de MHP.

Hipótesis 7: A partir de los estudios realizados por Nolen-Hoeksema (1994), los hombres utilizarán en mayor medida Estilos y Estrategias de Afrontamiento dirigidas a resolver el problema que las mujeres.

Hipótesis 8: Siguiendo los trabajos realizados por Guevremont et al. (1989); Bird y Harris (1990); Bolognini y Plancherel (1995); Copeland y Hess (1995); Zeidner (1995); Frydenberg et al. (1996); Bolognini et al. (1998); Frydenberg y Lewis (1991, 1993, 1999); Noam y Recklitis
(1999); Washburn (2000); Maslach y Reevy (2001), harán un mayor uso del Estilo de Afrontamiento Emocional las mujeres que los hombres.

Hipótesis 9. Partiendo de los resultados identificados en el estudio de Bermúdez et al. (2003), el Estilo de Afrontamiento Evitativo será en mayor medida utilizado por quienes se encuentren laboralmente inactivos en comparación a quienes trabajan.

Hipótesis 10. A partir de la información hallada por Bermúdez et al. (2003), los estudiantes laboralmente activos registrarán menores niveles de MHP que quienes no trabajan.

\section{Método}

\section{Participantes}

La muestra de estudiantes de este estudio estuvo compuesta por hombres y mujeres $(n=76)$, siendo 34 alumnos de 2do año y 42 alumnos de 4to año de un programa de grado universitario, fueron reclutados mediante un muestreo no-probabilístico, por conveniencia, es decir, las unidades fueron seleccionadas dada su conveniencia para el investigador y porque se pensó que es posible que representen más o menos a todo el universo (Morillo y Ruiz, 2004). Se estimó para este trabajo que el riesgo potencial de los participantes no era mayor que los beneficios.

Fueron considerados criterios de inclusión: (i) Ser estudiante a nivel universitario de grado que se encontraran cursando entre un $80 \%$ y un $100 \%$ de las materias pertenecientes a 2 do o 4to año, (ii) que la participación fuera voluntaria y confidencial.

Fueron considerados criterios de exclusión: (i) ser estudiante de algun otro nivel, (ii) Ser estudiante de alguna otra universidad.

A los efectos de evaluar los criterios de inclusión y exclusión indicados, fue considerada la Encuesta de datos socio-demográficos (elaborada ad hoc para este estudio). A través de la misma la información fue filtrada para que la muestra cumpliera con los criterios mencionados.

\section{Instrumentos de Medida}

Entre paréntesis se indica al valor de Alpha de Cronbach global de los distintos instrumentos, así como de las subescalas que los componen.

- Brief COPE, versión en español (Perczek, Carver, Pozo-Kaderman, \& Price, 2000) 
(a de Cronbach global =.78): Las Estrategias de Afrontamiento empleadas por los estudiantes fueron evaluadas utilizando el Inventario Brief COPE (Carver, 1997). Esta versión abreviada del instrumento original también es de autoinforme, consta de 24 ítems con un formato de respuesta tipo Likert de 4 puntos que van desde 1 "no hice esto en lo absoluto" a 4 "hice esto con mucha frecuencia". El motivo por el que los autores decidieron realizar una versión abreviada surgió a partir de la elevada frecuencia con que observaban respuestas de impaciencia desde sus pacientes, al completar el instrumento original completo de 60 ítems. Su índice de consistencia interna (Alpha de Cronbach) global es de .78. El instrumento se encuentra disponible en castellano, es de libre acceso y está autorizado para su uso según su autor, a través del sitio Web de University of Miami.

Para este estudio se utilizó la versión en castellano (Perczek et al., 2000). Cuando el autor seleccionó los ítems que formarían parte de la versión abreviada (Brief COPE, que tiene únicamente 2 ítems por escala), tuvo en cuenta los análisis factoriales previos, la claridad de los ítems y el grado en que los mismos eran significativos para los pacientes en un estudio previo (Carver, 1997). Del mismo modo, al crear un conjunto reducido de ítems, resultaron modificadas algunas de las escalas (Carver, 1997). Finalmente el Brief COPE en su versión en castellano quedó compuesto por las siguientes escalas: Afrontamiento activo ( $\alpha=.73)$; Planificación ( $\alpha=.58)$; Reinterpretación positiva ( $\alpha=.69$ ); Aceptación ( $\alpha=.66)$; Humor $(\alpha=.76)$; Religión $(\alpha=.86)$; Utilización de apoyo emocional $(\alpha=.81)$; Auto-distracción ( $\alpha=.51$ ); Negación ( $\alpha=.65)$; Expresión de emociones negativas $(\alpha=.47)$; Uso de sustancias $(\alpha=.50)$ y Desvinculación comportamental $(\alpha=.71)$.

Cabe mencionar que en la línea de investigación en Psicología de la Salud y Cáncer de Mama, dirigida por la Prof. Dra. Micaela Reich, se realizó el estudio de las propiedades psicométricas del Brief COPE (Reich, Costa y Remor, en prensa) para la población nacional así como el análisis factorial exploratorio del inventario. De esta manera, se utilizó el análisis factorial preliminar realizado en nuestro país, en una muestra de 180 personas sanas, para contrastar la factorización propuesta por Carver (1997) que arrojó como resultado la consideración de 9 Estrategias de Afrontamiento, agrupadas en tres Estilos: Afrontamiento por Evitación (AEV),
Afrontamiento Focalizado en el Problema (AFP) y Afrontamiento Focalizado en la Emoción (AFEM).

- Encuesta de datos socio-demográficos elaborada ad hoc: Para la recolección de datos socio-demográficos se utilizó una Encuesta de elaborada ad hoc para este estudio, que incluye información acerca de la edad, el género, el año de carrera que se encuentra cursando y actividad laboral.

- CASO-A30, (Caballo et al., 2010) (a de Cronbach global =.92): Se utilizó a demás el Cuestionario de Ansiedad Social para Adultos (CASO-A30, Caballo et al., 2010) compuesto por 30 ítems que evalúan el grado de malestar, tensión o nerviosismo que produce la interacción social en distintas situaciones para evaluar el MHP. Su índice de consistencia interna (Alpha de Cronbach) global es de .92. Este instrumento surgió como consecuencia de la escasez de parámetros definidos y unificados para juzgar cuando la Ansiedad Social era o no un problema generalizado. Sus autores observaron que la decisión solía estar basada en dos criterios antes que se elaborara la técnica. Uno de ellos consiste en el número de situaciones temidas y el otro en una elevada puntuación global en el instrumento utilizado, con lo que ambos criterios se tornan cuestionables, ya que dependía del instrumento que se utilizara y de su construcción, el tipo de variabilidad de las situaciones evaluadas que se obtendría. A partir de ello se construyó el CASO-A30, como un nuevo instrumento de evaluación de la Ansiedad Social, compuesto por 30 ítems que se puntúan en una escala tipo Likert de cinco puntos, desde 1= "Nada o muy poco malestar, tensión o nerviosismo" hasta 5 = "Mucho o muchísimo malestar, tensión o nerviosismo".

Este cuestionario evalúa cinco dimensiones de la Ansiedad Social: 1) Hablar en Público/ Interacción con personas de autoridad, 2) Interacción con desconocidos, 3) Interacción con el sexo opuesto, 4) Expresión asertiva de molestia, desagrado o enfado, y 5) Quedar en evidencia o en ridículo. Cada una de las dimensiones o factores consta de seis ítems distribuidos al azar a lo largo del cuestionario.

Cabe resaltar que en el trabajo realizado por Caballo et al. (2010) sobre el CASO-A30 con el objetivo de presentar las propiedades psicométricas del instrumento en una muestra de 15504 estudiantes universitarios de 20 carreras universitarias de 17 comunidades autónomas españolas, cuyas edades oscilaban entre los 17 
y 60 años, el 19, 25\% de los participantes eran estudiantes de la Licenciatura en Psicología, siendo la carrera que reunía mayor cantidad de estudiantes en la muestra.

Las conclusiones acerca de este trabajo realizado por Caballo et al. (2010), mencionan que es un cuestionario válido y fiable para su uso con toda la población universitaria española, así como que su evaluación de la Ansiedad por dimensiones resultó novedosa y más cercana a la realidad de las personas con Ansiedad Social, que los cuestionarios tradicionales utilizados hasta el momento.

\section{Procedimientos}

Una vez obtenida la aprobación del Comité de Ética de Investigación de la Universidad Católica del Uruguay, se acordó una fecha y un horario, se pasó a presentar las características generales de la investigación y el consentimiento informado a los participantes potenciales donde se mencionaba la participación voluntaria y anónima (Buki y Reich, 2010). Una vez hecha la introducción se les solicitó que llenaran el Inventario Brief COPE, que permite evaluar las Estrategias de Afrontamiento empleadas frente a estresores, para lo que se les pidió que respondieran pensando en situaciones en las que hubiesen tenido que Hablar en Público. Luego el cuestionario de Ansiedad Social para Adultos CASO-A30, que permitió obtener información referida al MHP.

Posteriormente, se les pidió que llenaran la encuesta diseñada ad hoc para obtener información sobre las variables socio-demográficas. Por último, se realizó un contrabalanceo sistemático de las medidas de las variables estudiadas, para reducir los efectos del orden, según lo establecen León y Montero (2005), el tiempo de evaluación fue de aproximadamente 15 a 20 minutos por alumno.

\section{Diseño}

Se condujo una investigación no experimental, ex post-facto, descriptiva, comparativa, correlacional y de corte transversal según la clasificación que indican León y Montero (2005).

\section{Análisis Estadístico}

Para la realización del análisis estadístico de los datos arrojados por las técnicas administradas, se utilizó el paquete estadístico SPSS versión 20.0 para Windows. Inicialmente se condujeron análisis de frecuencias y análisis descriptivos para representar los datos. De las escalas utilizadas, se obtuvieron estadísticos de normalidad, asimetría y curtosis.

Como medidas de resumen se calcularon las medias, mínimos y máximos, y desviaciones típicas. Se condujo un análisis correlacional de las variables continuas estudiadas, utilizando el coeficiente de Rho de Spearman, luego de haber realizado pruebas de normalidad y haber constatado un nivel de significatividad $p \leq .05$. Para hallar la normalidad de los tres Estilos y las cinco dimensiones de Ansiedad se llevaron a cabo pruebas Kolmogorov-Smirnov. La información recabada permitió determinar que la mayoría de las distribuciones no fueron normales, considerando estadísticamente significativo $p \leq .05$. para todos los conjuntos de datos. En este sentido decidimos utilizar pruebas paramétricas ( $T$ de Student) y no paramétricas ( $U$ de Mann-Whitney) en la comparación de medias. Para comparar la información referida a la edad agrupada por tramos utilizamos el ANOVA no paramétrico ( $\mathrm{H}$ de Kruskal-Wallis) por tratarse de más de dos grupos. Adicionalmente se condujeron análisis de contingencias para conocer la existencia estadística de asociación entre las variables categóricas.

\section{Resultados}

A continuación se exponen los principales resultados alcanzados a partir de este estudio, buscando dar respuesta a los objetivos anteriormente descritos. Las Tablas 1 y 2 resumen la información referida a las características sociodemográficas, laborales y acerca de las puntuaciones obtenidas en relación a las Estrategias y los Estilos de Afrontamiento.

En la Tabla 1 puede observarse que el mayor porcentaje de alumnos se encontraba en 4to, con una edad media de 23 años al momento del estudio $(n=76)$. Por otro lado, se puede apreciar que la mayoría de los participantes tenía entre 21 y 25 años; eran predominantemente mujeres, se encontraban laboralmente inactivos y cursando 4to. año. 
Análisis descriptivo de los instrumentos utilizados

En la Tabla 2 se observa que dentro de las subescalas o Estrategias de Afrontamiento que componen el Brief COPE, la más utilizada por los participantes para afrontar el MHP, fue Utilización de apoyo emocional, seguida de Planificación, mientras que las subescalas que recibieron puntuaciones más bajas en general

Tabla 1

Características socio-demográficas y laborales más salientes $(n=76)$; Estudiantes de los cursos de 2do. y 4to.

\begin{tabular}{|c|c|c|c|c|}
\hline \multirow{2}{*}{ Variables } & & \multicolumn{2}{|c|}{$\%$ Por grupo } & \multirow{2}{*}{$\begin{array}{c}\text { \% Total de la muestra } \\
\qquad(\mathrm{n}=76)\end{array}$} \\
\hline & & 2 do. & 4to. & \\
\hline Edad (años) M (DT) & & $21,59(3,44)$ & $24,37(5,68)$ & $23,06(4,92)$ \\
\hline \multirow[t]{4}{*}{ Edad (en tramos) } & $\begin{array}{l}\text { De } 19 \text { a } 20 \\
\text { años }\end{array}$ & 52,9 & 0 & 23,7 \\
\hline & $\begin{array}{l}\text { De } 21 \text { a } 25 \\
\text { años }\end{array}$ & 35,3 & 76,2 & 57,9 \\
\hline & 26 o más & 11,8 & 14,3 & 13,2 \\
\hline & Sin dato & 0 & 9,5 & 5,3 \\
\hline \multirow[t]{3}{*}{ Actividad Laboral } & Trabaja & 23,5 & 47,6 & 36,8 \\
\hline & No trabaja & 76,5 & 50 & 61,8 \\
\hline & Sin dato & 0 & 2,4 & 1,3 \\
\hline \multirow[t]{3}{*}{ Género } & Femenino & 85,3 & 90,5 & 88,2 \\
\hline & Masculino & 14,7 & 7,1 & 10,5 \\
\hline & Sin dato & 0 & 2,4 & 1,3 \\
\hline \multirow[t]{3}{*}{ Curso } & 2 do. & & & 44,7 \\
\hline & 4to. & & & 55,3 \\
\hline & Sin dato & & & 1,3 \\
\hline
\end{tabular}

Tabla 2

Medias y desviaciones típicas de las Estrategias y Estilos de Afrontamiento, Brief COPE $(n=76)$.

\begin{tabular}{lc}
\hline Estrategias & $\boldsymbol{M}(\boldsymbol{D T})$ \\
\hline Afrontamiento Activo & $5,92(1,252)$ \\
Planificación & $5,96(1,361)$ \\
Reinterpretación positiva & $5,88(1,575)$ \\
Aceptación & $5,75(1,462)$ \\
Humor & $4,68(1,805)$ \\
Utilización de apoyo emocional & $6,18(1,622)$ \\
Expresión de emociones negativas & $4,72(1,554)$ \\
Religión & $3,80(1,811)$ \\
Auto-distracción & $5,17(1,676)$ \\
Negación & $3,00(1,442)$ \\
Uso de sustancias & $2,36(0,905)$ \\
Desvinculación comportamental & $2,89(1,332)$ \\
AFP & $17,76(3,29)$ \\
AFEM & $21,34(4,20)$ \\
AEV & $17,22(4,42)$ \\
\hline
\end{tabular}


fueron Desvinculación comportamental y Uso de sustancias. Asimismo, puede observarse que de los tres Estilos de Afrontamiento que componen el instrumento, Afrontamiento Focalizado en la Emoción fue al que más recurrieron los participantes y Afrontamiento Evitativo el utilizado en la minoría de los casos, pero el que mayor dispersión presenta en sus puntuaciones.

Dentro de las dimensiones descritas en la Tabla 3 y que componen el CASO-A3O se puede apreciar que Quedar en Evidencia o en Ridículo representa el mayor promedio de Ansiedad asociada al MHP, siendo en segundo lugar Interacción con el Sexo Opuesto la dimensión que mayores grados de Ansiedad reportó, mientras que Interacción con Desconocidos alcanzó las puntuaciones más bajas en la muestra estudiada.

Tabla 3

Medias y desviaciones típicas de las dimensiones, CASO-A30 $(n=76)$

\begin{tabular}{lc}
\hline Dimensiones & $\boldsymbol{M}(\boldsymbol{D T})$ \\
\hline $\begin{array}{l}\text { Hablar en público, Interacción con personas } \\
\text { de autoridad }\end{array}$ & $16,79(5,066)$ \\
Interacción con desconocidos & $13,53(5,027)$ \\
Interacción con el sexo opuesto & $18,38(4,475)$ \\
Expresión asertiva de molestia, desagrado & $17,04(5,363)$ \\
y enfado & \\
Quedar en evidencia o en ridículo & $19,63(4,439)$ \\
Sumatoria del puntaje del CASO -A30 & $85,37(18,627)$ \\
\hline
\end{tabular}

\section{Análisis descriptivo de las variables socio- demográficas en relación al Afrontamiento y los niveles de Ansiedad}

A partir de la información provista en la Tabla 4 se pueden observar las interacciones de las distintas variables dependientes con las variables socio-demográficas.

En relación a los Estilos de Afrontamiento, se observa que AFP presentó una disminución en la frecuencia para Afrontar el MHP conforme aumentó la edad de los estudiantes, contrariamente a lo observado con AEV (a mayor edad, menor valor promedio).

En relación a la actividad laboral, no se encontraron grandes diferencias en cuanto a la preferencia por un Estilo, a excepción del Afrontamiento Evitativo que para aquellos laboralmente inactivos indicó una media $(M=18,0)$ y para los participantes laboralmente activos $(M=15,9)$, siendo de los 3 Estilos el que presentó mayores diferencias de acuerdo a la actividad laboral.

Se pudo observar que las mujeres registraron puntuaciones más elevadas en Afrontamiento Evitativo $(M=17,4)$ que los hombres $(M=16,3)$ y éstos utilizaron más el Afrontamiento Focalizado en el Problema y Focalizado en las Emociones ( $M=18,5$ y $M=22,1$, respectivamente) que las mujeres.

La mayor diferencia registrada al comparar los Estilos según el curso, fue la registrada en Afrontamiento Evitativo entre los estudiantes de 2do. $(M=19,8)$ y 4 to. $(M=15,1)$.

Las dimensiones del Cuestionario de Ansiedad Social para Adultos (CASO-A30) (Caballo et al. 2010) indicaron que Interacción con el Sexo Opuesto presentó diferencias según el género. Los hombres consideraron esta situación con menores niveles de $\operatorname{MHP}(M=16,3)$ que las mujeres $(M=18,5)$. Vinculando la misma dimensión con la edad, se identificó que quienes tenían más de 26 años registraron menores valores de MHP $(M=15,4)$, interpretándose como que hallaron menos ansiógenas las situaciones en las que debieran interactuar con personas del sexo opuesto los estudiantes de mayor edad. Sin embargo, Expresión Asertiva de Molestia presentó resultados inversos a los recién mencionados ya que conforme aumentaba la edad (19-20 $M=16,3 ; 21-25 M=17,0 ; 26$ o más $M=18,4$ ) se hallaron mayores valores lo que podrían interpretarse como que a más edad, más MHP producían aquellas situaciones en las que debieran comunicar en forma clara y precisa una molestia.

La situación de Hablar en Público obtuvo mayores puntuaciones entre los estudiantes que no trabajaban $(M=17,4)$ que entre los que sí lo hacían $(M=15,8)$; entre las mujeres $(M=16,9)$ que entre los hombres $(M=15,8)$; entre los alumnos de 2 do. $(M=17,9)$ que entre los de 4to $(M=15,9)$, indicando que a excepción de la edad, el resto de las variables socio-demográficas (género, actividad laboral y el curso) contribuirían a distinguir niveles diferenciales de MHP en la muestra estudiada.

Interacción con Desconocidos obtuvo mayores puntuaciones entre los estudiantes que no trabajaban $(M=14,0)$ que los que sí lo hacían $(M=12,6)$.

La dimensión Quedar en Evidencia o en Ridículo marcó una leve diferencia entre hombres 
Tabla 4

Medias y desvios típicos de las dimensiones de Ansiedad, Estrategias y Estilos de Afrontamiento $(n=76)$ comparados por variables socio-demográficas

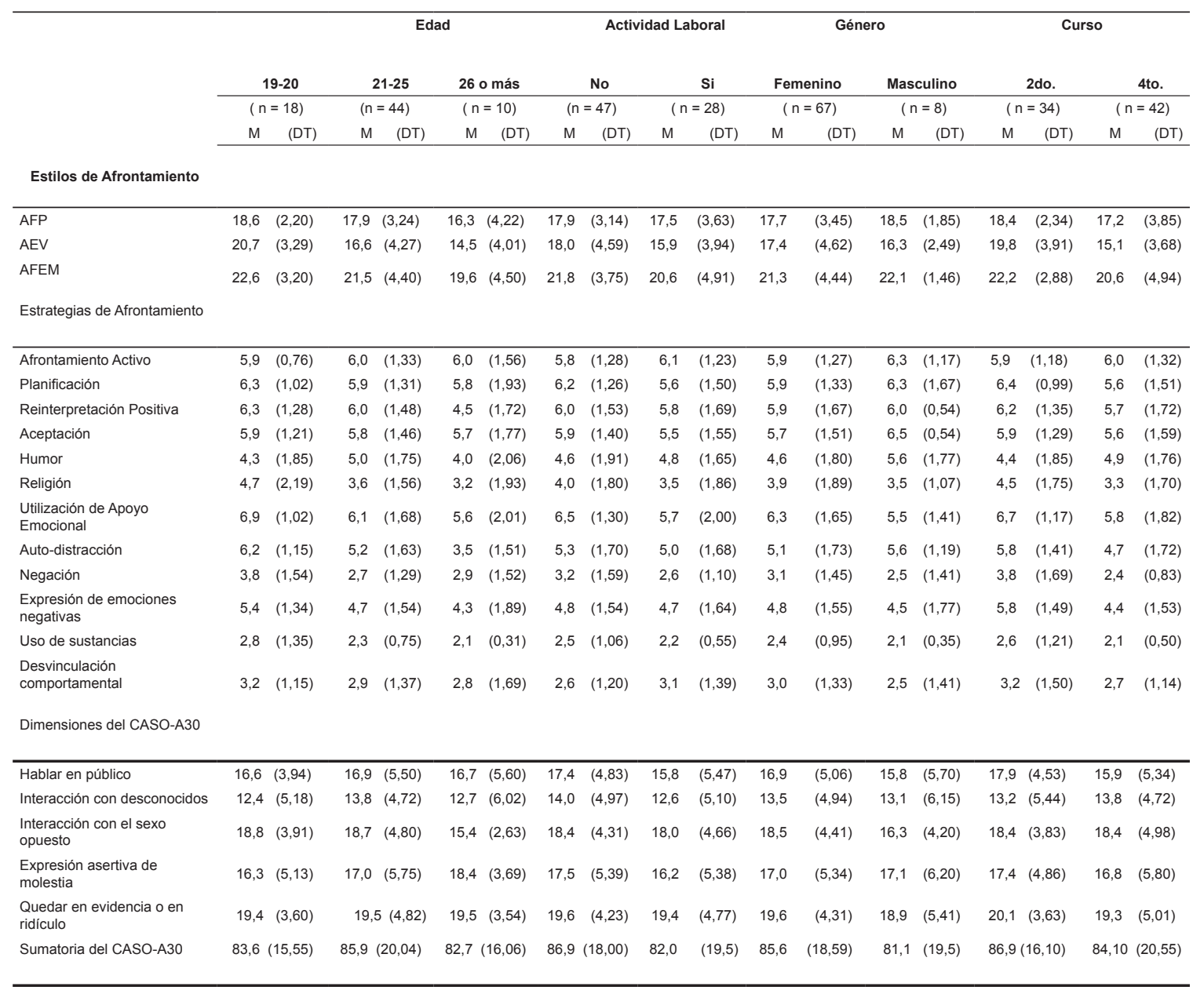

$(M=18,9)$ y mujeres $(M=19,6)$, así como entre 2do $(M=20,1)$ y 4to $(M=19,3)$, interpretándose que tanto para las mujeres como para los estudiantes de 2do, este tipo de situaciones podría conllevar mayores niveles de ansiedad en relación a los hombres y a los alumnos de 4to.

\section{Asociaciones de las Estrategias, Estilos, dimensiones de Ansiedad y Edad}

A continuación, se presenta el estudios correlacional de las variables continuas analizadas, para lo cual se utilizó el coeficiente de Rho de Spearman, luego de haber realizado pruebas de normalidad, con un nivel de significatividad $p \leq .05$.

La Tabla 5 refleja las correlaciones significativas halladas entre las distintas variables.
Por ejemplo, el Estilo AEV $\left(r=-0.400^{* *}\right)$, la dimensión Interacción con el Sexo Opuesto $\left(r=-0.238^{*}\right)$ y las Estrategias Reinterpretación Positiva $\left(r=-0.260^{*}\right)$, Religión $\left(r=-0.238^{*}\right)$ y Autodistracción $\left(r=-0,413^{* *}\right)$ han correlacionado negativamente con la edad, indicando que cuanto menor edad tenían los estudiantes mayor la tendencia a utilizar el distanciamiento mental o conductual para afrontar el MHP, mayores niveles de Ansiedad les producirían situaciones en las que debieran interactuar con personas del sexo opuesto y mayor probabilidad de Afrontar el MHP valorando los estresores desde una perspectiva optimista, refugiándose en la religión como apoyo emocional y tomando distancia, concentrándose en otros proyectos. 


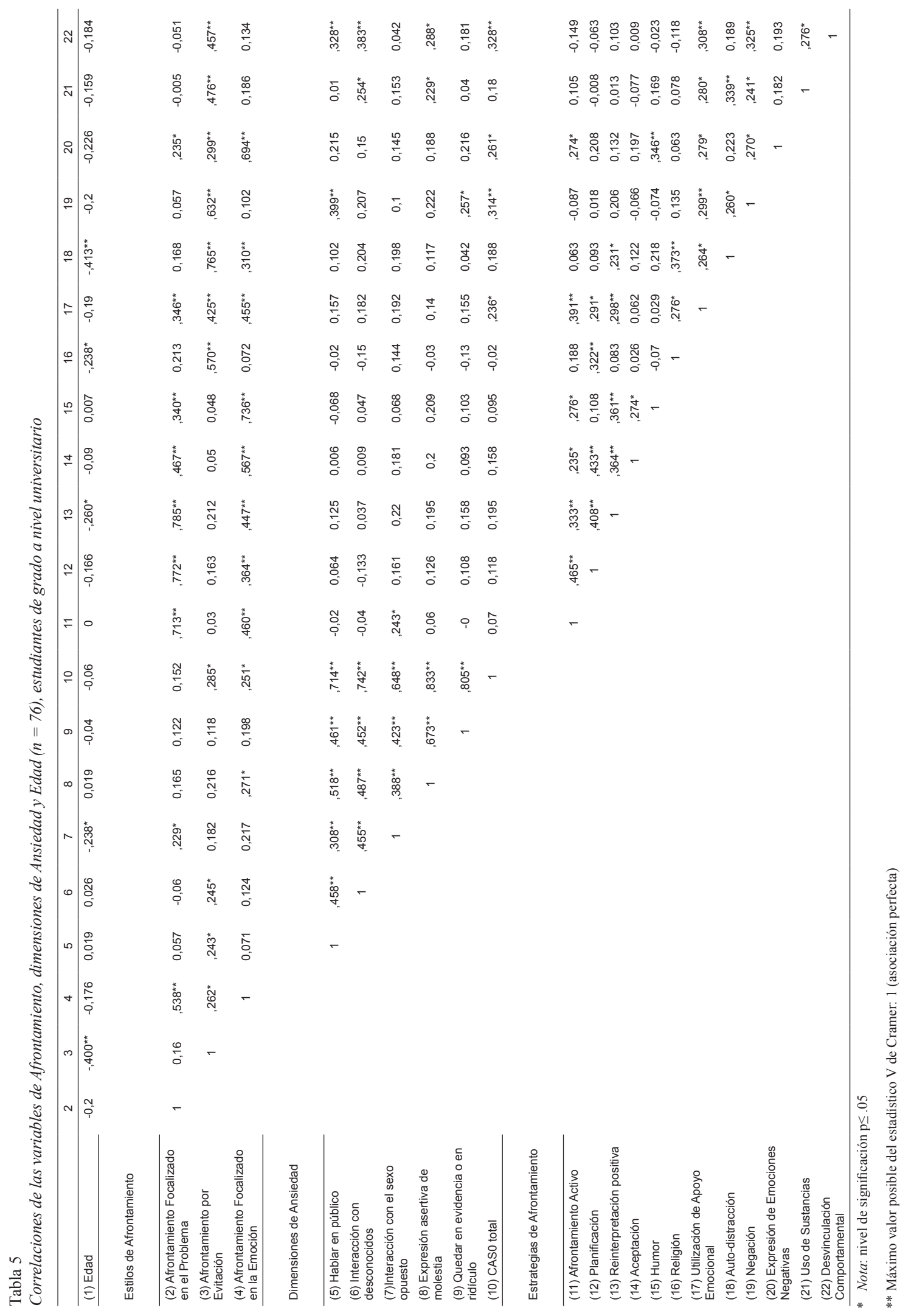


El Estilo de Afrontamiento Focalizado en el Problema (AFP) correlacionó positivamente con la dimensión Interacción con el Sexo Opuesto $\left(r=0.229^{*}\right)$ lo que según Carver et al. (1993); Folkman y Lazarus, (1984) se podría interpretar indicando que aquellos estudiantes que utilizaban un conjunto de Estrategias más dirigidas a resolver el problema y a suprimir las actividades distractoras como Afrontamiento activo, Planificación, Reinterpretación positiva y Aceptación, coincidirían con aquellos que más interacción con el sexo opuesto buscan tener pese al MHP. De las dimensiones de Ansiedad que recoge el CASO-A30, Hablar en Público $\left(r=0.243^{*}\right)$, Interacción con Desconocidos $\left(r=0.245^{*}\right)$ y CASO-A30 Total $\left(r=0.285^{\star}\right)$ correlacionaron positivamente con $\mathrm{AEV}$, lo que según Brennan, Holahan, Holahan, Moos y Schutte (2005), indicaría que aquellos estudiantes a quienes las situaciones de exposición ante un público y tener que interactuar con personas desconocidas les significara afrontar un estresor, presentarían una tendencia a distanciarse tanto mental como conductualmente y negar el evento estresante utilizando el humor para ello. También se deduce de estas correlaciones que cuanto mayores sean los niveles de Ansiedad total que indica el CASO-A3O frente al MHP, mayor es la probabilidad que el estudiante utilice un Estilo Evitativo para afrontarlo.

El Estilo de Afrontamiento Focalizado en la Emoción (AFEM) también correlacionó positivamente con el total del CASO-A30 $\left(r=0.251^{*}\right)$ y con la dimensión Expresión Asertiva de Molestia Desagrado y Enfado $\left(r=0.271^{*}\right)$ lo que según Folkman y Lazarus (1984); Carver, Scheier y Wientraub (1989); Admiraal, Korthagen y Wubbels (2000); podría estar indicando que para aquellos estudiantes a quienes expresar en forma clara y asertiva la molestia les representara valores elevados de MHP tendrían una tendencia a elegir Estrategias de Afrontamiento como la Utilización de apoyo emocional, Expresión de emociones negativas, Aceptación y Humor. La Estrategia de Afrontamiento activo correlacionó positivamente con la dimensión Interacción con el Sexo Opuesto $\left(r=0.243^{*}\right)$ indicando que los alumnos que se centran en conocer los pasos necesarios para eliminar la fuente del MHP manifestarían una tendencia a interactuar con personas del género opuesto.

La dimensión Quedar en Evidencia o en Ridículo correlacionó positivamente con la Es- trategia Negación $\left(r=0.257^{\star}\right)$ lo que indicaría que a más MHP manifestado por los alumnos al quedar en evidencia, mayor sería la tendencia a rechazar la realidad del problema en una primera valoración de la situación.

En relación a la puntuación total del CASOA30 se encontró que correlacionó positivamente con Utilización de apoyo emocional $\left(r=0.236^{*}\right)$, Negación $\left(r=0.314^{* *}\right)$, Expresión de emociones negativas $\left(r=0.261^{*}\right)$ y Desvinculación comportamental $\left(r=0.328^{* *}\right)$, lo que estaría indicando que a mayor puntuación total alcanzada por los estudiantes frente al MHP, mayor es la tendencia a elegir Estrategias de Afrontamiento evitativas o centradas en la emoción y con ello, menor la tendencia a centrarse en el problema para resolverlo.

Si bien no se encontró una correlación entre la edad y la Desvinculación comportamental $(p=.12, r=-0.184)$, ni con la Utilización de apoyo emocional $(p=.10, r=-0.194)$, ni con la Expresión de emociones negativas $(p=.06$, $r=-0.226$ ), ni con los niveles de Ansiedad total del CASO-A30, se podría decir que hay cierta asociación inversa o negativa, por lo que podría pensarse que a más edad, menor la tendencia de los estudiantes de elegir Estilos de Afrontamiento Evitativos o Focalizados en la Emoción para afrontar el MHP, así como también menores los niveles de Ansiedad que les produciría Hablar en Público.

Adicionalmente, se condujeron análisis de contingencias para estudiar la posible asociación entre las variables categóricas. Las variables género y curso no presentaron una asociación estadísticamente significativa entre sí (Razón de Verosimilitud $=0.30$ ); es decir, tanto el género femenino como el masculino indicaron ser variables que no se encontrarían asociadas al año que cursaban los participantes. Las variables género y trabajo tampoco presentaron asociación estadística significativa (Razón de Verosimilitud $=0.13$ ), indicando que la presencia o ausencia de actividad laboral no estaría asociada al hecho de ser hombre o mujer.

Las variables trabajo y curso mostraron una asociación estadística significativa entre sí (Razón de Verosimilitud $=0.04$ ) con una magnitud moderada ( $\mathrm{V}$ de Cramer $=0.279)$, encontrándose los estudiantes de 4to laboralmente activos en mayor medida que los de 2 do. Lo mismo sucede con las variables edad y curso, entre ellas se encontró una asociación estadísticamente significativa (Razón de 
Verosimilitud $=0.00$ ) con una magnitud fuerte $(V$ de Cramer $=0.640)$ que se traduce al decir que la edad podría estar asociada significativamente al curso en la carrera, dadas las características curriculares que exigen comenzar con edades más cercanas a los 19-20 años y en 4to. tener por tanto más de 20 años como lo indican los porcentajes de los distintos tramos etarios.

\section{Discusión}

El Estilo Focalizado en la Emoción fue el que se utilizó en mayor medida por los estudiantes. Por su parte, Utilización de apoyo emocional fue la Estrategia que alcanzó mayores puntuaciones medias. Una posible explicación a estos resultados podría radicar en la composición de la muestra, predominantemente femenina y según Guevremont et al. (1989); Bird y Harris (1990); Frydenberg y Lewis (1991, 1993, 1999); Bolognini y Plancherel (1995); Copeland y Hess (1995); Zeidner (1995); Bolognini et al. (1998); Frydenberg et al. (1996); Noam y Recklitis (1999); Washburn (2000) y Maslach y Reevy (2001), las mujeres suelen utilizar Estrategias de Afrontamiento centradas en la emoción en mayor medida que los hombres, quienes evidenciarían una tendencia a elegir Estrategias de Afrontamiento más activas y dirigidas a solucionar el problema, Nolen-Hoeksema (1994).

Al comparar el uso que hicieron ambos grupos de los Estilos de Afrontamiento, las frecuencias encontradas indicaron que los alumnos de 2do. utilizaron con mayor frecuencia el Estilo de Afrontamiento Evitativo (AEV) que los de 4to. Este Estilo correlacionó negativamente con la edad, con la dimensión Interacción con el Sexo Opuesto y con Estrategias como Reinterpretación Positiva, Religión y Autodistracción. Esto podría estar indicando que cuanto menor edad tenían los estudiantes, mayor la tendencia a utilizar el distanciamiento mental o conductual para afrontar el MHP, mayores niveles de Ansiedad les producirian las situaciones en las que debieran interactuar con personas del sexo opuesto y mayor probabilidad de Afrontar el MHP valorando los estresores desde una perspectiva optimista, refugiándose en la religión como apoyo emocional y tomando distancia, o concentrándose en otros proyectos.

De hecho, la diferencia del uso entre ambos grupos del Afrontamiento Evitativo (AEV) fue la más significativa y evidente. Heinrich y Spielberg (1982) y Casullo y Fernández (2001) sugieren como explicación a esta correlación, que el nivel de estudios alcanzado correlaciona negativamente con Estrategias centradas en la Emoción o Evitativas y por el contrario se asocia fuertemente con Estrategias focalizados en resolver el problema o modificar el estresor. Sin embargo, al comparar las frecuencias entre los grupos, en relación al Estilo Focalizado en el Problema (AFP) no fueron halladas diferencias significativas entre los estudiantes de 2do y 4 to. Contrariamente a lo establecido por Contini et al. (2009) y a nuestra hipótesis, se observó un incremento en el uso de este Estilo para los estudiantes de 2 do. en relación a que los de 4 to. Esta diferencia no es significativa y ello podría deberse al pequeño número de participantes que compusieron la muestra total y a cada grupo (34 alumnos en 2 do y 42 en 4 to).

De acuerdo a lo sugerido por Casullo y Fernández (2001), una mayor edad marcaría una tendencia a elegir Estrategias y Estilos de Afrontamiento focalizados en resolver el problema (AFP). Sin embargo, al comparar ambos grupos se pudo observar que a medida que aumentaba la edad, se mantuvo el uso del AFP entre los tramos etarios, entre los hombres y las mujeres, incluso entre los laboralmente activos o no, con variaciones mínimas que no alcanzaron la significación, por lo que se acepta parcialmente la hipótesis al respecto.

Entendemos oportuno considerar, ante este desencuentro entre lo propuesto por Casullo y Fernández (2001) y los resultados que arrojan nuestros datos, que si bien la tendencia hallada por ellos se vincula con una elección de Estrategias avocadas a resolver el problema conforme aumenta la edad, para nuestra investigación los rangos etarios son muy próximas entre sí. Este dato podría explicar en cierta medida la tendencia observada al comparar los Estilos de Afrontamiento, dividiendo a la muestra en grupos de edad de manera electiva. En este sentido, se observó que AFP y AFEM se mantuvieron próximos en sus promedios, sin mayores diferenciaciones entre los tres grupos etarios, a excepción del Afrontamiento Evitativo (AEV), que presentó una correlación negativa con la edad.

Una lectura de la asociación inversa o negativa de las variables que no llegaron a arrojar correlaciones estadísticamente significativas podría llegar a señalar que a mayor edad, menores niveles de Ansiedad y menor uso de Estilos centrados en la emoción o por evitación 
del estresor, tal como lo indica la literatura científica al respecto. No obstante, el tamaño de la muestra podría dificultar el hallazgo de significación estadística de las asociaciones como para registrase una correlación negativa entre la edad y AFEM, o como lo sugiere la literatura, mayor uso de AFP.

De las dimensiones de Ansiedad que recoge el CASO-A30, Hablar en Público, Interacción con Desconocidos y la sumatoria de las puntuaciones (CASO-A30 Total) correlacionaron positivamente con $A E V$, indicando que aquellos estudiantes a quienes las situaciones de exposición ante un público y tener que interactuar con personas desconocidas les significara afrontar un estresor, presentarían una tendencia a distanciarse tanto mental como conductualmente y negar el evento estresante, utilizando el humor para ello, de acuerdo a lo sugerido por Brennan et al. (2005). Asimismo, se desprende de estas correlaciones que cuanto mayores sean los niveles de Ansiedad total (CASO-A30 Total) mayor es la probabilidad de que el estudiante utilice un Estilo Evitativo para afrontar el MHP.

El Estilo de Afrontamiento Focalizado en el Problema (AFP) correlacionó positivamente con la dimensión Interacción con el Sexo Opuesto, lo que según Carver et al. (1993) y Folkman y Lazarus (1984) podría indicar que aquellos estudiantes que utilizaban un conjunto de Estrategias dirigidas a resolver el problema y a suprimir las actividades distractoras como Afrontamiento activo, Planificación, Reinterpretación Positiva y Aceptación, coincidirían con aquellos que más interacción con el sexo opuesto buscan pese al MHP que ello les pueda significar.

En cuanto a la incidencia de variables socio-demográficas, al comparar por género los niveles MHP se observaron diferencias que ubicarían a las mujeres con un mayor promedio que los hombres como lo sugirieron Bados (1992); Forde et al. (1996); Fernández-Turrado et al. (2005) y Caballo et al. (2010). Este dato, al igual que un mayor uso del Estilo Focalizado en la Emoción utilizado por las mujeres (si bien en nuestro estudio no se evidenció, pero tampoco se hallaron diferencias significativas en relación al uso que hacen de AFEM los hombres), podría ser interpretado a la luz de la información científica recabada. Podría pensarse que las mujeres manifestarían mayores niveles de MHP que los hombres dado que frente a estresores evidenciarían una tendencia a focalizarse en la Emoción -a diferencia de los hombres que recurrirían a Estrategias de Afrontamiento más activas y centradas en resolver el problema(Nolen-Hoeksema, 1994). Los hombres hicieron un mayor uso del Afrontamiento Focalizado en el Problema, confirmándose de esta manera las hipótesis mencionadas al respecto. Sin embargo, no fueron halladas diferencias que hablen de un mayor uso del Afrontamiento Focalizado en la Emoción entre las mujeres como lo sugieren Guevremont et al. (1989); Bird y Harris (1990); Frydenberg y Lewis (1991, 1993, 1999); Bolognini y Plancherel (1995); Copeland y Hess (1995); Zeidner (1995); Bolognini et al. (1998); Frydenberg et al. (1996); Noam y Recklitis (1999); Washburn (2000) y Maslach y Reevy (2001), aunque sí puntuaciones con mayores desviaciones típicas, mientras que los hombres registraron desviaciones típicas más concentradas. Observando las correlaciones, se puede apreciar que AFEM también correlacionó positivamente con el total del CASO-A30 y con la dimensión Expresión Asertiva de Molestia Desagrado y Enfado. Lo anterior, de acuerdo con Folkman y Lazarus (1984); Carver et al. (1989); Admiraal et al. (2000), indicaría que para aquellos estudiantes a quienes expresar en forma clara y asertiva las molestias les implica niveles elevados de MHP, tendrían una tendencia a elegir Estrategias de Afrontamiento como la Utilización de apoyo emocional, Expresión de emociones negativas, Aceptación y Humor.

Al observar el uso que hicieron los estudiantes laboralmente activos del Afrontamiento Evitativo, tal como lo sugieren Bermúdez et al. (2003) se evidenció que los estudiantes sin actividad laboral utilizaban en mayor medida este Estilo para Afrontar el MHP lo que podría interpretarse en base a las referencias científicas existentes al respecto, que señalan que la actividad laboral estaría asociada a la construcción de la imagen que las personas tienen de sí mismas, a los niveles de satisfacción y bienestar personales. Estaría además asociada a la tendencia a tomar una postura activa ante las situaciones estresantes como consecuencia de un mayor grado de control y eficacia percibidos frente al estresor. Asimismo, se observaron menores niveles de MHP entre los estudiantes laboralmente activos, lo que podría estar indicando que estos percibirían un mayor nivel de control sobre la situación estresante que los laboralmente inactivos, apoyando de esta manera las hipótesis en relación a la actividad laboral y sus efectos sobre el uso del Afrontamiento 
Evitativo. Por otro lado, podemos agregar la asociación encontrada con el análisis de contingencia e interpretarlo sugiriendo que cursar 2do. o 4to. estaría asociado con el aumento o disminución de actividad laboral y con ello, a diferencias en los niveles de MHP reportados.

En cuanto a las variables de Ansiedad, uno de los datos hallados al comparar entre grupos la sumatoria del CASO-A30 y observar los niveles de Ansiedad según los distintos tramos etarios, indicó menores niveles de Ansiedad conforme aumenta la edad, en la dirección de lo encontrado por Argyle (1981); Brown y Harris, (1982); Heinrich y Spielberg (1982), Bird et al. (1987); Bland et al. (1988); Fyer et al. (1992); Angst \& Degonda (1993); Davidson et al. (1993); Echeburúa (1993); Elhers et al. (1995) Bersabé et al. (1996); Forde et al. (1996); Bascarán et al. (1999); Alfano et al. (2001); Hidalgo et al. (2002); Caballo et al. (2003); Fernández-Turrado et al. (2005). Sin embargo, si bien la adolescencia temprana sería el período en que suelen aparecer las manifestaciones más evidentes y luego a medida que aumenta la edad disminuirían los niveles de Ansiedad, en nuestro estudio aparece una mayor concentración de Ansiedad en el tramo de 21 a 25 años, disminuyendo hacia los 26 o más. Este dato es consistente con la información sugerida por Caraveo y Colmenares (2000), que identificaron que si bien para los hombres la adolescencia es el punto de mayor expresión de MHP, las mujeres alcanzan los mismos niveles posteriormente en torno a los 24 años, edad comprendida en el tramo donde aparecieron mayores niveles de Ansiedad, considerando que nuestra muestra está compuesta en su mayoría por mujeres. Al comparar entre tramos etarios los niveles de MHP si bien no se encontraron diferencias estadísticamente significativas, se observaron mayores niveles de Ansiedad entre los estudiantes de 19 o 20 años al comparar las puntuaciones totales obtenidas con el CASO-A30. Se observó una asociación negativa que podría estar indicando que a los estudiantes de menor edad las situaciones de Hablar en Público les significarían mayores niveles de Ansiedad que al resto.

En la línea de los sugerido por Heinrich y Spielberg (1982), podríamos interpretar estos datos considerando que la Ansiedad descendería a medida que aumenta el nivel de escolaridad y la edad, Asimismo, otra de las hipótesis contrastadas y apoyadas en relación a lo propuesto por Heinrich y Spielberg (1982) y Casullo y Fernández (2001), es la relativa a los niveles de MHP por curso. Los mayores niveles observados entre los estudiantes de 2do. podrían estar asociados al hecho de que la Ansiedad perjudica menos a medida que aumenta la escolaridad de las personas, como lo sugieren estos autores en sus estudios.

En relación a las dimensiones del CASOA30, Quedar en Evidencia o en Ridículo al Hablar en Público fue la que alcanzó mayores puntuaciones para el total de la muestra, como en los grupos, si bien para los estudiantes de 2do. fue levemente más ansiógena que para los de 4to. Esto podría estar indicando en la misma dirección que lo mencionado por Heinrich y Spielberg (1982), Argyle (1981); Brown y Harris (1982); Bird et al. (1987); Bland et al. (1988); Fyer et al. (1992); Angst \& Degonda (1993); Davidson et al.(1993); Echeburúa (1993); Elhers et al. (1995) Bersabé et al. (1996); Forde et al.( 1996); Bascarán et al. (1999); Alfano et al.(2001); Casullo y Fernández (2001) Hidalgo et al. (2002); Caballo et al.(2003); FernándezTurrado et al. (2005), que encontraron que conforme aumenta la edad y el nivel de escolarización, menores serían las manifestaciones de Ansiedad o MHP registradas.

La Estrategia de Afrontamiento activo correlacionó positivamente con la dimensión Interacción con el Sexo Opuesto indicando que los alumnos centrados en conocer los pasos necesarios para eliminar la fuente del MHP manifestarían una tendencia a interactuar con personas del género opuesto.

El CASO-A30 Total correlacionó positivamente con Utilización de apoyo emocional, Negación, Expresión de emociones negativas y Desvinculación comportamental, lo que estaría indicando que a mayores niveles de MHP, mayor es la tendencia a elegir Estrategias de Afrontamiento evitativas o centradas en la emoción y con ello, menor la tendencia a centrarse en el problema para resolverlo.

La literatura existente en relación al Afrontamiento nos impulsa a reflexionar respecto a la plasticidad, la flexibilidad y diversidad de Estrategias asociadas a la salud. Los estudios más recientes indican cada vez más que es la plasticidad de respuestas, capacidad de adaptación y selección de la Estrategia de Afrontamiento según el estresor y los recursos con que cuenta la persona en ése momento, lo que se asocia a mayor salud mental. Como opuesto a ello, la rigidez en las posibilidades al 
momento de afrontar un estresor. De este modo, la Estrategia Activa puede ser adaptativa ante determinados estresores o circunstancias, y no otras, como cuando necesitamos hacer un Afrontamiento de tipo emocional, por ejemplo. En esta dirección, Steptoe (2000) menciona que desde la perspectiva transaccional se puede deducir la existencia de distintas respuestas en dos personas ante una misma situación, incluso en la misma persona frente a distintas circunstancias. Moore (2002) propone que algunas personas reaccionarán frente a una situación adversa con altos niveles de amenaza considerándola un desafío, aun sin disponer de recursos para Afrontar la misma, poniéndose en marcha para tomar control de la situacion.

Consideramos como fortaleza de la presente investigación haber podido evaluar un grupo de estudiantes universitarios en el contexto de Uruguay y que hasta la fecha no se haya publicado ningún trabajo con estas características a nivel nacional según nuestro conocimiento.

EI MHP constituye un problema frecuente que afecta tanto alumnos universitarios como profesores y población en general (Henderson \& Pollard, 1988). Como consecuencia, se ven afectadas tareas recurrentes en la población universitaria que pueden afectar negativamente la futura vida profesional de los estudiantes (Bados, 1992; Forde et al., 1996; Alvarado et al., 2000).

Se apuntó a lograr una aproximación y caracterización de la muestra que ofrezca a los interesados en el tema de Afrontamiento ante el MHP un aporte a partir del cual, sería interesante continuar trabajando.

Entre las limitaciones que más se destacan de la presente investigación, consideramos el tamaño y la composición de la muestra. La muestra estuvo compuesta mayoritariamente por mujeres, con edades comprendidas entre los 21 y los 25 años, laboralmente inactivas y en su mayoría estudiantes de 4to. Ello indica que los resultados identificados podrían diferir si la muestra hubiese sido más heterogénea en relación a la actividad laboral, el género, el curso y la edad. Esto limita la generalizabilidad de nuestros resultados hacia estudiantes de género masculino, de otras carreras, y universidades diferentes de nuestro medio. Entendemos sería interesante continuar investigando acerca de los procesos de Afrontamiento de los estudiantes universitarios ante el MHP.

En relación a las características de la muestra, el tamaño y su composición no per- miten generalizar los datos obtenidos a todos los estudiantes a nivel de grado universitario. El diseño transversal implica la imposibilidad de obtener evidencia longitudinal así como de establecer relaciones causales entre las variables.

Cabe destacar que el muestreo por conveniencia utilizado en esta investigación, a través del cual se reclutó a los participantes en los salones de los correspondientes cursos, podría afectar en cierta medida los resultados, ya que la participación de los estudiantes en este estudio, podría verse afectada por factores como la experiencia en relación al MHP de cada estudiante, la asistencia a clase el día del reclutamiento y el número de participantes.

Otra de las limitaciones consiste en que el cuerpo de literatura existente sobre la evaluación del Afrontamiento ha crecido en gran medida en las últimas tres décadas, y si bien son claros los principios que pueden derivarse de esta literatura en su conjunto, la idea de que hay aún muchas preguntas sin respuesta es casi unánime (Carver, 1997).

Se plantea como sugerencia para estudios futuros en relación al Afrontamiento de universitarios delMHP, que la muestra sea mayor. Ello permitiría, entre otras cuestiones, aproximarnos al estudio de las propiedades psicométricas y análisis factoriales de los instrumentos seleccionados para la muestra estudiada.

Se sugiere además que la muestra se componga de mayor proporción de hombres, observar si las diferencias arrojadas por los resultados marcan algún tipo de agrupación etaria que se caracterice en forma similar a cómo se describe en la literatura y que se tenga en cuenta la heterogeneidad de la actividad laboral.

Teniendo en cuenta los factores de riesgo, se observa que las mujeres de 2 do. año, sin actividad laboral, con edades comprendidas entre 21 y 25 años fueron quienes reportaron mayores niveles de MHP y por lo tanto podrían considerarse la población más vulnerable. Una sugerencia para futuras intervenciones podría vincularse al entrenamiento en habilidades sociales, incluso entrenamientos que incluyan debates y ejemplificaciones de diferentes Estrategias de Afrontamiento frente a diferentes estresores, posibilitando la ampliación del repertorio individual e incorporación de un mejor conocimiento sobre las herramientas propias al momento de discernir fuentes de Estrés y resultados.

No obstante, y a modo de conclusión, los resultados aquí presentados son alentadores, 
y en resumen, suman información a la aproximación y caracterización de este colectivo, observando el Afrontamiento ante el MHP de los estudiantes, destacándose la relevancia y necesidad de continuar con el estudio de estas variables, atendiendo aquellos aspectos y criterios mencionados previamente como limitaciones.

\section{Referencias}

Admiraal, W. F., Korthagen, F. A. J., \& Wubbels, T. (2000). Effects of student teachers' coping behaviour. British Journal of Educational Psychology, 70(1), 33-52.

Alfano, C. A., Beidel, D. C., Ferrell, C., \& Yeganeh, R. (2001). The treatment of childhood social anxiety disorder. Psychiatric Clinics of North America, 24, 831-846.

Alvarado, J. M., García, M.V. y Jiménez, A. (2000). La predicción del rendimiento académico: regresión lineal versus regresión logística. Psicothema, 2, 248-252.

Angst, J., \& Degonda, M. (1993). The Zurich Study XX. Social phobia and agoraphobia. European Archives of Psychiatry and Clinical Neuroscience, 243, 95-102.

Argyle, M. (1981). The contriution of social interaction research to social skills training. En J. D. Wine \& M. D. Smye (Eds.) Social Competence, 261-286. Ney York: Guildford Press.

Bados, A. (1991). Hablar en público: Guía práctica para lograr habilidad y confianza. Madrid: Pirámide.

Bados, A. (1992). Análisis de componentes de un tratamiento cognitivo-somático-conductual del miedo a hablar en público. (Tesis doctoral microfichada original de 1986). Barcelona: Publicaciones de la Universidad de Barcelona.

Bados, A. (2001). Habilidades de enfrentamiento al Estrés: Intervención para hablar en público. En J. M. Buceta, A. M. Bueno y B. Más. (Eds.), Intervención psicológica y salud: Control del Estrés y conductas de riesgo (pp. 167-214). Madrid: Dykinson.

Bados, A. (2008). Miedo a hablaren público. Manuscrito inédito, Departament de Personalitat, avaluació i Tractament Psicológics, Universitat de Barcelona, España.

Bascarán, M. T., Iglesias, C., Jiménez, L. y Sáiz, P. J. (1999). Epidemiología del Trastorno por Ansiedad Social. En J. Bobes, González, M.P., Sáiz, P. A. \& Bousoño, M. (Eds.), Abordaje actual del Trastorno por Ansiedad Social (pp. 3- 14). Barcelona: Masson.

Beck, A. T. (1976). Cognitive therapy and emocional disorders. Madison, C. T.: International University Press.

Beatty, M. J., \& Valencic (2000). Context-based apprehension vs. planning demands: A communibiological analysis of anticipatory public speaking anxiety. Communication Education, 49, 57-71.

Bermúdez, J., Ciccottelli, H., García, J., Gascón, S. y Olmedo, M. (2003). Estrés por desempleo y salud. Cuadernos de medicina psicosomática y psiquiatría de enlace, 66, 9-17.

Bersabé, R. M., Bragado, C., Carrasco, I. y Sánchez, M. L. (1996). Trastornos de Ansiedad en escolares de 6 a 17 años. Ansiedad y Estrés, 2, 97-112.

Betson, C., Lam, T., Stewart, S., Wong, A., \& Wong, C. (1999). A Prospective Analysis of Stress and Academic Performance in the First Two Years of Medical School. Med Educ, 33, 243-50.

Bird, G. W., \& Harris, R. L. (1990). A comparison of role strain and coping strategies by gender and family structure among early adolescents. Journal of Early Adolescence, 10, 141-158.
Bird, H. R., Bravo, M., Canino, G. J., Guevara, L. M., RubioStipec, M., Martínez, R., ...Shrout, P.E., (1987). The prevalence of specific psychiatric disorders in Puerto Rico. Archives of General Psychiatry, 44, 727-735.

Bland, R. C., Newman, S. C., \& Orn, H. (1988). Lifetime prevalence of psychiatric disorders in Edmonton. Acta Psychiatrica Scandinavica, 77, 24-32.

Bolognini, M., \& Plancherel, B. (1995). Coping and mental health in early adolescence. Journal of Adolescence, 18, 459-474.

Bolognini, M., Halfon, O., \& Plancherel, B. (1998). Coping strategies in early and mid-adolescence: Differences according to age and gender in a community sample. Europe and Psychologist, 3(3), 192-201.

Booth-Butterfierld, S. (1988). Instructional interventions for reducing situational anxiety and avoidance. Communication Education, 37, 214-223.

Brown, R. D., \& Harris, K. R. (1982). Cognitive behavior modification and informed teacher treatment shy children. Journal of Experimental Education, 50, 137-143.

Borkovec, T. D., \& Lane, T. W. (1984). The influence of therapeutic expectancy/demand on self-efficacy ratings. Cognitive Therapy and Research, 8, 95-106.

Brennan, P. L., Holahan, C. J., Holahan, C. K., Moos, R. H., \& Schutte, K. K. (2005). Stress Generation, Avoidance Coping, and Depressive Symptoms: A 10-Year Model. Journal of Consulting and Clinical Psychology, 73(4), 658-666.

Buki, L. y Reich, M. (2010). Directrices para la Utilización de Cuestionarios. Proyecto: Aspectos Culturales del Cáncer en Mujeres Uruguayas. Manuscrito inédito, Departamento de Ciencias Cognitivas y de la Salud, Universidad Católica del Uruguay.

Caballo, V. E., Arias, B., Equipo de Investigación CISO-A, Irurtia, Ma. J. y Salazar, I. C. (2010). Validación del "Cuestionario de Ansiedad Social para adultos" (CASO-A30) en universitarios españoles: similitudes y diferencias entre carreras universitarias y comunidades autónomas. Psicología Conductual, 18(1), 5-34.

Caballo, V. E., García-López, L. J., López-Gollonet, C., Olivares, J. y Rosa, A. I. (2003). Una revisión de los estudios epidemiológicos sobre fobia social en población infantil, adolescente y adulta. Psicología Conductual, 11, 405-428.

Caraveo, J. y Colmenares, E. (2000). Prevalencia de los trastornos de ansiedad fóbica en la población adulta de la ciudad de México. Salud Mental 23(5), 1-10.

Carver, C. S. (1997). You want to measure coping but your protocol's too long: Consider the Brief COPE. International Journal of Behavioral Medicine, 4, 92-100.

Carver, C. S., Scheier, M. F., \& Wientraub, J. K. (1989), Assessing coping strategies: A theoretically based approach. Journal of personality and social psychology, 56(2), 267-283.

Carver, C. S., Harris, S. D., Noriega, V., Robinson, D. S., Scheier, M. F., Pozo, C.,...Clark, K.C.(1993). How coping mediates the effect of optimism on distress: A study of women with early stage breast cancer. Journal of Personality and Social Psychology, 65, 375390.

Casullo, M. M. y Fernández Liporace, M. (2001). Estrategias de Afrontamiento en estudiantes adolescentes. Investigaciones en Psicología, 6(1), 25 - 49.

Clark, D. M., \& Wells, A. (1995). A cognitive model of social phobia. En R. Heimberg, M. Liebowitz, D. A. Hope \& F. R. Schneier (Eds.), Social Phobia: Diagnosis, Assessment and Treatment, 69-93. New York: Guilford Press. 
Contini, N., Fernández, M., De la Iglesia, G., Organato, P. y Savedra, E., (2009). Estrategias de Afrontamiento frente a problemas académicos en estudiantes medios y universitarios, Revista Iberoamericana de Diagnóstico y Evaluación Psicológica (RIDEP), 27(1), 63-84.

Copeland E. P., \& Hess, R. S. (1995). Differences in young adolescents ' coping strategies based on gender and ethnicity. Journal of Early Adolescence, 15, 203-219.

Davidson, J. R.T., Ford, S.M., Krishnan, K. R. R, Potts, N. L. S., Richichi, E., Smith, R. D., Wilson, W.H. (1993). Treatment of social phobia with clonazepam and placebo. Journal of Clinical Psychopharmacology, 13, 423-429.

D’El Rey, G. J. F. y Pacini, C. A. (2005). Medo de falar em público em uma amostra da populaça: prevalencia, impacto no funcionamento pessoal e tratamento. Psicología: Teoría e Pesquisa, 21, 237-242.

Duarte, I. B., Finch, C., Hoffmann P., Kelly, L., Keaten, J. A., \& Michels, M. M. (2002). Family communication patterns and the development of reticence. Communication Education, 51, 281-285.

Echeburúa, E. (1993). Fobia Social. Barcelona: Martínez Roca.

Ehlers, A., Hofmann, S. G., \& Roth, W.T. (1995). Conditioning therapy: a model for the etiology of public speaking anxiety. Behaviour Research and Therapy, 33, 367-371.

Ellis, K. (1995). Apprehension, self-perceived competency and teacher immediacy in the laboratory-supported public speaking course: trends and relationship. Communication Education, 50, 357-373.

Fernández-Abascal, E. G. (1997). Estilos y Estrategias de Afrontamiento. En E. G. Fernández-Abascal, F. Palermo, M.Chóliz y F. Martínez (Eds.), Cuadernos de práctica de motivación y emoción (pp. 189-206). Madrid. Pirámide.

Fernández-Turrado, T., Herrero, Ma. L., Orejudo, S., Nuño, J. y Ramos, T. (2005). El desarrollo de la competencia para hablar en público en el aula a través de la reducción de la ansiedad ante esta situación. Estudio previo. Revista Electrónica Interuniversitaria de Formación del Profesorado, 8 (1), 1-6.

Fisher, S. (1984). Stress and perception of control. London: Lawrence Erlbaum.

Fisher, S. (1994). Stress in academic life. Mental assembly line. Buckingham: Society for Research into Higher Eductation.

Folkman, S., \& Lazarus R. S. (1984). Stress, appraisal, and coping. New York: Springer-Verlag.

Forde D. R., Stein, M. B., \& Walker, J. R. (1996). Publicspeaking fears in a community sample: Prevalence, impact on functioning and diagnostic classification. Archives of General Psychiatry, 53, 169-174.

Fortney, S. D., Jonson, D. I., \& Long, K. M. (2001). The impact of compulsive communicators on the selfpercived competence of classroom peers: An investigation and test of instructional strategies. Communication Education, 50, 357-373.

Frydenberg, E., \& Lewis, R. (1991). Adolescent coping: the different ways in wich boys and girls cope. Journal of Adolescence, 14, 119-133.

Frydenberg, E., \& Lewis, R. (1993). Boys play sport and girls turn to others: age, gender and ethnicity as detrerminants of coping. Journal of Adolescence, 16, 252-266.

Frydenberg, E., \& Lewis, R. (1999). Things don't better just because you're older: A case for facilitating reflection. British Journal of Educational Psychology, 69, 81-94.

Frydenberg, E., Parsons, A., \& Poole, C. (1996). Overachievement and coping strategies in adolescent males. British Journal of Educational psychology, 66, 109-114.
Fyer, A. J., Gorman, J. M., Martin, L. Y., Liebowitz, M. R., \& Schneier, F. R. (1992). Alcohol abuse in social phobia. Journal of Anxiety Disorder, 3, 15-23.

Guevremont, D. C. Spirino, A., Stark , L. J., \& Williams, C. A. (1989). Common problems and coping strategies I: Findings with normal adolescents. Journal of Abnormal and Child Psychology, 17(2), 203-212.

Gutiérrez, M. (1996). Ansiedad y deterioro cognitivo: incidencia en el rendimiento académico. Ansiedad y Estrés, 2, 173-94.

Henderson J. G., \& Pollard C. A. (1988). Four types of social phobia in a community sample. The Journal of Nervous and Mental Discase, 176, 440-445.

Heinrich, D.L. \& Spielberger, C.D. (1982). Anxiety and complex learning. In H.W. Krohne \& L. Laux (Eds.), Achievement, stress and anxiety (pp.145-165). Washington, DC: Hemisphere.

Hernández, J. M., Polo, A. y Pozo, C. (1996). Evaluación del Estrés académico en estudiantes universitarios. Ansiedad y Estrés, 2, (2-3), 159-172.

Hidalgo, N. D., Inglés, C. J. y Méndez, F. X. (2002). Estrés en las relaciones Interpersonales: Un estudio descriptivo en la adolescencia. Ansiedad y Estrés, 8, 25-36.

Keaten J. A., \& Kelly, L. (2000). Treating communication anxiety: Implications of the communibiological paradigm. Communication Education, 49, 45-57.

King, P. E., \& Smith, C. D. (2004). Students feedback sensitivity and the efficacy of feedback interventions in public speaking performance improvement. Communication Education, 53, 203-216.

Kozak, M. J., Lang, P. J., Levin, D. N., \& Mliller, G. A. (1983). Fear behavior, fear imaginery and the psychophysiology of emotion: The problem of affective response integration. Journal of Abnormal Psychology, 92, 276-306.

Lazarus, R. S. (2000). Stress and Emotion. A New Synthesis. Nueva York: Springer Publishing Company.

León O. G. y Montero, I. (2005). Sistema de clasificación del método en los informes de investigación en Psicología. International Journal of Clinical and Health Psychology, 5, (1), 115-127.

Martin, M. M., Mottet, T. P., \& Myers, S. A. (2004). Relationships among perceived instructor verbal approach and avoidance. Relational strategies and students' motives for communicating with their instructors, Communication Education, 53, 116-122.

Maslach, C., \& Reevy, G. M. (2001). Use of social support: Gender and personality differences. Sex Roles, 44, 437-459.

McAnintrye P. D., \& McDonald, J. R. (1998). Public speaking anxiety: Perceived competence and audience congeniality. Communication Education, 47, 359-365.

Moore, K. (2002). Positive Psychology and Health: Situational Dependence and Personal striving. En E. Frydenberg (Comp.), Beyond Coping. Meeting goals, visions, and challenges (pp. 107-125). Nueva York: Oxford University Press.

Morillo, L. y Ruiz, A. (2004). Epidemiología Clínica. Investigación clínica aplicada. Bogotá: Editorial Médica Panamericana.

Noam G. G. \& Recklitis, C. J. (1999). Clinical and developmental perspectives on adolescent coping. Child Psychiatry and Human Development, 30(2), 87-101.

Nolen-Hoeksema, S. (1994). An interactive model for the emergence of gender differences in depression in adolescence. Journal of Research on Adolescence, $4,519-534$. 
Perczek, R., Carver, C. S., Pozo-Kaderman, C., \& Price, A.A., (2000). Coping, mood, and aspects of personality in Spanish translation and evidence of convergence with English versions. Journal of Personality Assessment, 74(1), 63-87.

Reich, M., Costa, D. y Remor, E. (en prensa). Estudio de las Propiedades Psicométricas del Brief-COPE para una muestra de mujeres uruguayas.
Steptoe, A. (2000). Stress, social support and cardiovascular activity the working day. International Journal of Psychophysiology, 37(3), 299-308.

Washburn, J. M. (2000). The influence of gender, sex-role orientation, and self-esteem on adolescent's use of coping strategies. Dissertation Abstracts International Section A; Humanities and Social Sciences, 61(1A), 88.

Zeidner, M. (1995). Adaptive coping with test situations:Areview of the literature. Educational Psychologist, 30, 123-133.

Para citar este artículo:

Maldonado, I. y Reich, M. (2013). Estrategias de afrontamiento y miedo a hablar en público en estudiantes universitarios a nivel de grado. Ciencias Psicológicas VII (2): 165 - 182. 